2017-06

\title{
Multimedia Communications in Internet of Things QoT or QoE?
}

\author{
Karaadi, A
}

http://hdl.handle.net/10026.1/12534

10.1109/ithings-greencom-cpscom-smartdata.2017.11

2017 IEEE International Conference on Internet of Things (iThings) and IEEE Green Computing and Communications (GreenCom) and IEEE Cyber, Physical and Social Computing (CPSCom) and IEEE Smart Data (SmartData)

IEEE

All content in PEARL is protected by copyright law. Author manuscripts are made available in accordance with publisher policies. Please cite only the published version using the details provided on the item record or document. In the absence of an open licence (e.g. Creative Commons), permissions for further reuse of content should be sought from the publisher or author. 


\title{
Multimedia Communications in Internet of Things QoT or QoE?
}

\author{
Amulya Karaadi, Lingfen Sun, Is-Haka Mkwawa \\ School of Computing, Electronics and Mathematics \\ University of Plymouth \\ Plymouth, PL4 8AA, United Kingdom \\ Email:\{Amulya.Karaadi,L.sun,Is - Haka.Mkwawa\}@plymouth.ac.uk
}

\begin{abstract}
Multimedia communications play a major role in Internet of Things (IoT) applications such as traffic control and management, environmental monitoring, healthcare, surveillance, event recognition and home monitoring and automation. To ensure the quality of multimedia content to be collected, processed and delivered appropriately in such applications, it is necessary to design and develop a quality aware IoT architecture for multimedia IoT applications. Research on Quality of Experience (QoE) in multimedia communications in IoT has gained attention in recent years. However, the concept of IoT is to connect everything in a smart way without human interaction. Therefore, this paper introduces a new concept of Quality of Things (QoT) for multimedia communications in IoT, a new architecture based on the QoT for multimedia communications in IoTs is also proposed together with its challenges and future research directions.
\end{abstract}

Keywords-Quality of Things (QoT); Quality of Experience (QoE); Internet of Things (IoT); Multimedia Communications in Internet of Things (MIoT);

\section{INTRODUCTION}

The Internet of Things (IoT) is a novel network paradigm, which has gained great momentum in recent years. The IoT is formed up by billions of smart objects and/or smart devices connected by the Internet. According to Statista [1], the number of IoTs reached over 22 billion in 2016 and is expected to reach 50 billion by 2020 . The smart objects and/or devices such as Radio Frequency Identification tags (RFID), sensors, actuators, controllers, smart phones and smart TVs are able to communicate and interact with each other via heterogeneous wireless networks (e.g., WI-FI and Blue-tooth), in this way the objects or things are managed, identified and controlled [2]. The applications of IoT have reached transportation, education, healthcare, home environment and automation, smart home and smart cities. The IoT objects can collect, process and deliver a variety of information ranging from simple scalar data (e.g. ping message to indicate whether a device is alive) to rich multimedia contents such as audio, image and video.

Multimedia wireless sensor networks which process huge multimedia traffic in real time IoT applications such as traffic monitoring, remote system monitoring and home security monitoring, smart grid monitoring require huge memory and computational resources and consume more energy compared with traditional wireless sensor networks collecting information from the physical environment (e.g., light, pressure and temperature). According to the recent forecast of the Cisco Visual Networking Index [3], global IP traffic will increase nearly threefold from 2015 to 2020, and IP video traffic will be about $82 \%$ by 2020 .

Multimedia communications in real time IoT applications may experience network delay and congestions due to bandwidth constraints and packet loss, which have an adverse impact on the delivered multimedia quality. QoE/QoS aware Multimedia communications in IoTs (MIoT) have been proposed in recent years [4], [5] to address the above issues. However, according to Qualinet QoE is the overall acceptability of an application or service perceived by the end user, and is more user-driven [6]. Accordingly, the concept of IoT is the connection of number of devices/objects that communicate in a smart way without human involvement. For example, CCTV monitoring in real time IoT application, the quality of images (e.g., car number plate, accidents and traffic jam) should be detectable by detection software. In this context we do not need to consider QoE. So, are the current QoE models for multimedia communications in IoTs still appropriate? In general, QoE is a term to represent the end user experience for a multimedia service (e.g., video streaming or on-line video gaming). It is used for services with customers as the consumption entity. However, in IoT concepts, machine to machine (M2M) communications will be the dominant applications. With the billions of things/objects connected by the internet, the QoT concept is more appropriate than the concept of QoE because in most cases, human beings are not the ultimate target. To the best of the authors knowledge this is the first paper discussing the concept of QoT for multimedia communications in IoTs.

In this paper, the new concept of QoT for multimedia applications in IoTs is introduced together with its architecture based on the QoT for MIoTs applications is proposed which takes different quality of things/objects metrics into account for successful IoT application. The rest of the paper is organized as follows: In section II, basic IoT architecture is presented. In section III, multimedia applications of IoT and enabling technologies are discussed. In section IV, the concept of QoT for MIoTs is discussed. In Section V, QoT aware MIoTs based on cross layer architecture model is proposed. In section VI challenges and future research directions are discussed and the conclusion is given in Section VII. 


\section{IOT ARCHITECTURE}

The Internet of Things (IoT) is an integration of a large number of heterogeneous objects or devices with different computing and connecting capabilities which can harvest the information from the physical world connected by the internet. Traditional IoT architecture proposed in [7], is based on a layered model which has 3 layers

1) Physical level layer: the main function of this layer is to acquire the physical information of an environment with different scalar devices and then transmit the data to the network layer via the wireless sensor network (WSN) gateway.

2) Network Layer:the network layer fuses all the information and sends it to upper layers through the internet or wireless network.

3) Application Layer: this layer is a combination of service layer and application layer, service layer stores the information from the network layer, it provides decision making, data analysis and information management. Application layer provides services to different IoT applications, the typical applications include intelligent logistics, precision agriculture and smart city.

This model mainly focuses on the sensing, actuating and networking capabilities. However, the challenges posed by the multimedia communications in IoT devices are ignored. Authors in [8] have defined MIoT in 3 scenarios based on the use of multimedia content such as multimedia as IoT Input and output, multimedia as IoT input and multimedia as IoT output, and proposed a QoE layered model for MIoT applications, which has 5 layers:

1) Real-World Layer: Real world objects such as physical multimedia objects or devices acquire the multimedia content from the environment and send it to the virtual object layer through the network layer.

2) Network Layer: this layer considers traditional QoS parameters such as delay, packet loss and jitter can measure the performance of data transmission.

3) Virtualization Layer: in this layer, virtual objects are created to virtualize the functionalities of the real-world objects or devices.

4) Combination Layer: in this layer, composite virtual objects are created. It is composed of different virtual objects to provide determined service, that a single virtual object cant accomplish.

5) Application Layer:this layer considers the needs of an application and is mainly focused on QoE evaluation and control in terms of control, interactivity and presentation.

\section{MIoT AND ENABLING TeChNOLOGIES}

The MIoT is an emerging paradigm which has been used in many applications, we summarize some key multimedia IoT applications and enabling technologies below:

\section{A. Applications of MIoT}

MIoT have been discussed in previous studies [4], [9]] but their contribution and integration into research are still in early stages. However there are some applications that came into practice [5], [10], [11]. We have divided such applications as traffic monitoring, environment monitoring, home security monitoring, smart surveillance and e-health monitoring.

1) Traffic Monitoring: This domain application is largely related to monitor the traffic in real time scenarios, such as vehicles and roads equipped with video sensors (cameras), actuators providing important up-to-date information to the users for better navigation, so that the user can choose the congestion free route. On the other hand, government departments such as police, fire and ambulance would also receive accurate information about road accidents and traffic jams.

2) Environment Monitoring: Recently the availability of inexpensive hardware such as sky video cameras that are able to sense the information from the cloud environment and predict the environment conditions for the next 48 hours, enable grid power suppliers to receive accurate information about sun light and wind power.

3) Security Home Monitoring: CCTV cameras and motion sensors in smart home environment are able to identify thefts or illegal entrance into homes and activates an alarm when an event occurs so that the user can be alerted and take certain action according to that situation.

4) Smart surveillance: Video streams in multimedia internet of things enable public safety, for example if an attack happens on a crowded street the smart multimedia devices such as video sensors and cameras deployed in such environments can detect the attack event and report it to near by hospitals for emergency medical support. Simultaneously, the attacker can also be identified based on the moment of the attacker. The camera controller will adjust their resolutions and continuously provide updates of the attacker's location, meanwhile the controller will pass the information to identify attacker criminal database, police or medical database to check the attackers previous address.

5) E-health monitoring:Real-time patient health monitoring can be done by the doctors or nurses with the support of special sensors such as blood pressure and thermometer connected through the internet via wireless gateway. For example various IoT sensors/objects measure the patient's medical conditions such as temperature, blood pressure, pulse, heart beat and display the status in a visual format such as animations and graphs.

\section{B. Enabling Technologies for MIoT}

In this section, we discuss four enabling technologies (i.e., Wireless Sensor Networks, Cloud Computing, M2M, and $\mathrm{H} 2 \mathrm{M})$ towards MIoTs.

1) Wireless sensor networks (WSN): Deploying WSN for MIoTs applications is gaining attraction from the both academia and the industry in areas such as home/building energy monitoring and environment monitoring. Using WSN including camera sensors and actuator sensors that can sense scalar data such as tem- 
perature, pressure, humidity and multimedia information from the surrounding environment can improve the efficiency, reliability and safety of a MIoTs application. For example wireless multimedia sensors are able to monitor the renewable energy sources such as sun/wind intensity and direction, and predict the information. Also realtime multimedia monitoring system such as CCTV can be used to monitor critical aspects [12].

2) Cloud Computing: Cloud computing is a service that is PaaS (Platform as a Service), IaaS (Infrastructure as a service) and SaaS (Software as a service). CC (cloud computing) offers different resources (scalable computing, storage capacity). Users can monitor and control devices at any time and anywhere. Recently fog/edge computing is a popular method to handle delay sensitive MIoT applications [13].

3) Machine to Machine $(M 2 M)$ : This refers to the multimedia communication between two or more devices (machines) without any human interaction. For example in traffic control system there are camera sensors used to monitor variables such as traffic speed, accidents, and road congestions. There is detection software used to send all the information across the computers that controls the traffic by showing traffic lights and signs. IEEE, IETF and ETSI standardization activities going on M2M communications [14], [15].

4) Machine to Human (M2H): This refers to multimedia communication between machine to human. The main $\mathrm{M} 2 \mathrm{H}$ application is E-health monitoring, which is an application/service where patients can be monitored remotely by doctors, smart sensors collect patient information and present that information in a multimedia format so that doctors could get information about patient health. Another application of $\mathrm{M} 2 \mathrm{H}$ is navigation system, which is a service where roads equipped with camera sensors provide accurate information (such as traffic delays) to the end-user, so that the user could select a better route.

\section{QOT FOR MIOT}

To ensure the quality of multimedia content such as audio, video, and image to be collected, processed and delivered in MIoT applications it is necessary to design and develop a quality aware IoT architecture. Few studies have discussed the concept of quality aware IoTs. In [7], [8], [16] the authors focused on QoS/QoE metrics to increase the customer satisfaction for MIoTs applications. However, M2M communications will be the dominant applications in IoT [14], [15] and the basic concept of IoT is to connect the number of objects/devices in a smart way without human involvement, in such cases QoE is not applicable. Traditional QoS parameters only considered network parameters such as delay and jitter, packet loss and ignore other factors such as environmental factors and device factors. Here the concept of QoT comes into consideration for M2M communications in MIoTs. In general, QoT means Quality of Things, it is a successful operation of
IoT system. It focuses on the quality of multimedia data to be collected, processed and delivered between two or more devices/objects in an IoT environment. The goal of the QoT is to meet minimum quality that an IoT object can meet the minimum requirement of an IoT application. By meeting the minimum requirements of an IoT object on an IoT application, the system will avoid over provisioning of multimedia quality and hence, use less bandwidth. It is also envisaged that the system will save the energy in an IoT objects.

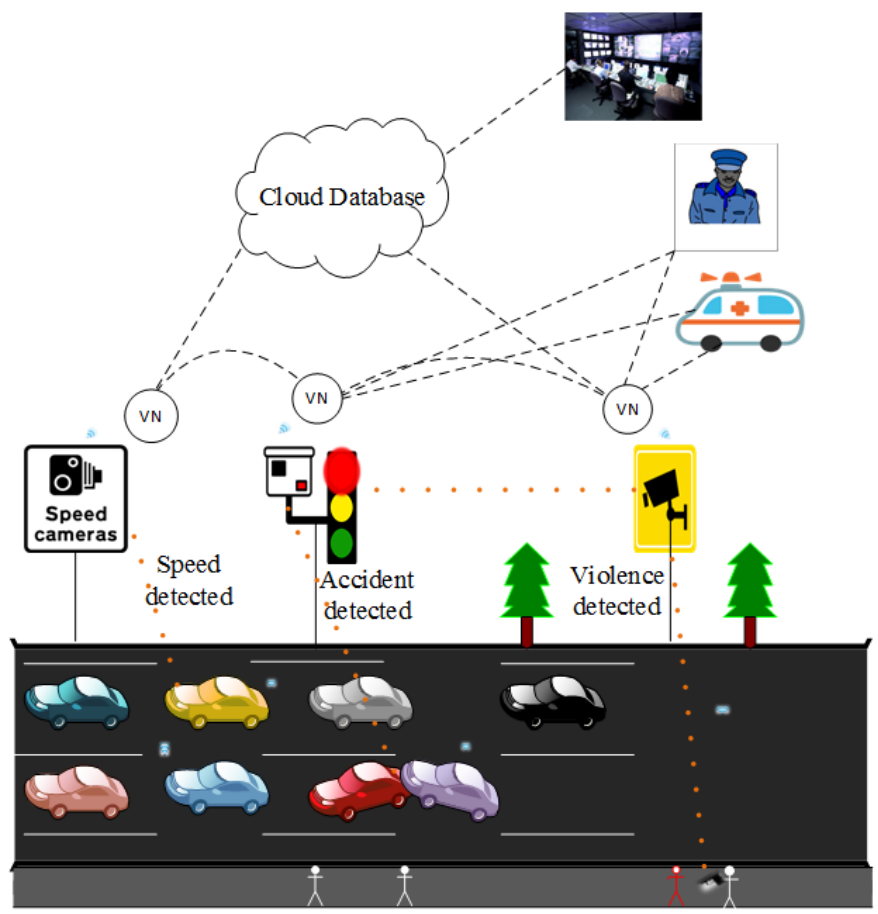

Fig. 1. QoT in MIoT

Fig. 1 illustrates the scenario of QoT for MIoTs with several IoT system devices such as camera sensors, scalar sensors are deployed in an IoT environment to monitor real-time traffic environment. Conventionally, the attacks or accidents are discovered by multimedia streaming to remote server or pre-processing video tasks at the camera nodes. However, the above two concepts would lead to various delays; processing video tasks at the camera node may lead to computational delays due to limited computational resources, and delivering video tasks to the remote cloud database may lead to network delays and traffic congestions due to limited bandwidth. Conversely, in QoT for MIoT applications the process can be done at virtual nodes to interpret the accidents or human violence and appropriate approaches can be followed such as reporting to health service department or the police department.

The scenario in Fig. 1, is a real-time traffic monitoring system. A speed camera deployed on a road detects the car registration number and sends the information to a virtual object to check the owners details such as name and home address for further processing. If an accident occurs in an IoT environment, the camera sensor can detect the event and send the information to virtual nodes. The virtual nodes 
process the information and report to a nearby police station. If the person is critically injured it sends an alert message to ambulance centre. Similarly, a gun shot fired by an attacker can be detected by the camera sensor which can send a report to virtual nodes to process the information and report to ambulance service and police service, and at the same time the information will be sent to other traffic signals to control the traffic or alert the crowd on the street. The attacker would be identified by his/her behaviour amongst the crowd. Virtual nodes send this information to the cloud to check the attackers previous records such as criminal history. The quality of images and videos captured by the camera sensors should meet the requirement to be able to be detectable by a detection software for further investigation. Here the QoT metrics play an important role to improve the connection between device to device or machine to machine. For $\mathrm{M} 2 \mathrm{H}$ communications such as E-health monitoring and navigation systems, it takes the QoE metrics into consideration such as end-user devices, preferences, satisfaction, and background.

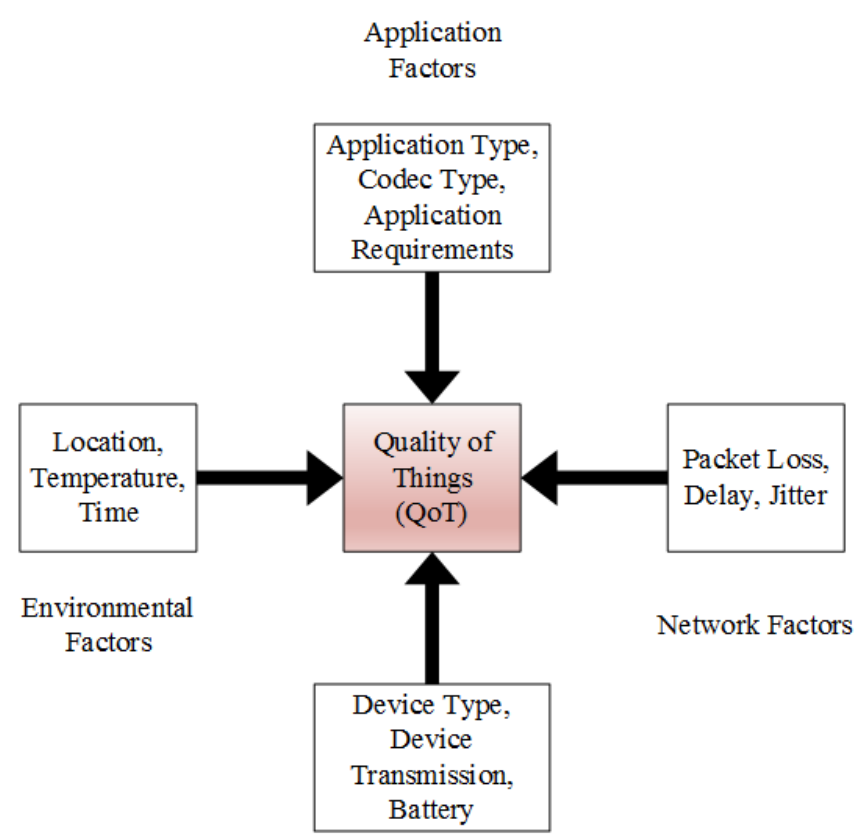

Device Factors

Fig. 2. The elements of QoT for MIoTs applications

Fig. 2, describes the main QoT factors for MIoTs applications that can affect service/application performance which includes monitoring, data collection, processing and delivery. These factors are described as follows,

1) Environmental Factors: The factors that can affect the QoT requirements on environment such as physical location, temperature and accuracy of time for an IoT application . For example, the information or data captured by the multimedia devices sent to the network gateway needs to measure the accuracy of the information such as physical location, time and temperature for further processing.

2) Device Factors: Real-time multimedia monitoring in an IoT platform may lead to high energy. Thus, device factors such as device type, device transmission, battery can affect the device life time according to the current device state.

3) Network Factors: Network factors such as packet loss, and jitter have an impact on the network performance. For instance, packet loss will cause data loss and thus, affect the quality of data transmission.

4) Application Factors: Application factors such as application and codec types are focused to meet the requirement of an MIoT device. For example, codec type such as $\mathrm{H} 265$ could be used instead of $\mathrm{H} 264$ to minimize network bandwidth at the same time providing high quality video transmission.

\section{QOT ARCHITECTURE}

Before proposing a QoT architecture based on cross layer model for MIoT, we identify some challenges that will affect the design and implementation of the QoT models.

\section{A. Challenges on QoT modelling}

We summarize some challenges in modelling the QoT for multimedia communications in IoT applications as follows.

1) Heterogeneity: MIoT devices have different functionalities, computational power and resolutions. Thus, one of the enabling IoT factors is the integration of number of devices and communications. So how to provide QoT for Heterogeneous multimedia IoT devices is a challenging task.

2) Scalability: integration of WSN in IoT opens a new horizon for many networking applications. The number of IoT devices connecting to the internet is growing rapidly. Those devices produce huge amount of data. Thus, providing QoT for increasing large applications is another challenge.

3) Quality Management: for M2M communications quality management is very challenging due to object/thing mobility in real time environments. For example, in real time traffic monitoring scenarios objects such as moving vehicles and humans, cameras deployed in such environments should be able to detect car number plates in motion. In such cases quality management is very important. For M2H communications it takes QoE factors into considerations. So how to develop QoT model by taking considerations of $\mathrm{QoE}$ for $\mathrm{M} 2 \mathrm{H}$ communications is another challenge.

4) Intelligence: once MIoT devices are deployed in a realtime environment its services and functionalities should be automatic and dynamic according to that situation. Building intelligence in such objects to operate adaptively without human involvement is another challenge. 


\section{B. Proposed QoT model}

As mentioned in the introduction, the aim of our work is to introduce a concept of QoT and propose a QoT architecture for MIoT applications. To support different applications in MIoT and to satisfy the quality requirements, the proposed architecture needs to address the above challenges, our new QoT model for MIoTs applications which is based on a cross layered IoT architecture is composed of different quality factors such as environmental factors, device factors, network factors and application factors. Recently adoption of cross layer model [17], [18] in multimedia communications in IoTs mainly focused on QoS parameters. Modelling QoT based on cross layer model is an interesting part. Traditional layered architectures are divided into different layers with different functionalities with only sharing information between adjacent layers and there is no direct communication between nonadjacent layers. Cross layer model solves the issues and provide a direct communication between different layers (nonadjacent layers). Thus, we proposed QoT architecture based on a cross layer model so that, the quality of information provided by the MIoT devices/things can be shared to different layers in order to improve the performance of the IoT system based on its current state.

Fig. 3 represents a proposed QoT model based on cross layered architecture. It takes into consideration things/device and network perspectives. It also considers user perspective (if a human being is involved in that application). It is composed of 5 layers: physical, network, virtual, service and application layers. We describe the layers in detail in the following paragraphs.

1) Physical Level Layer: this is the first layer in our proposed framework model, where heterogeneous physical multimedia devices/objects are deployed in an IoT environment to collect the information from the physical world. The devices can communicate with each other via wireless sensor networks. The multimedia content collected by an IoT device is sent to the virtual layer via heterogeneous wireless networks such as wifi, cellular network and blue-tooth. This layer mainly focuses on the QoT factors such as environmental and device factors. For example traffic monitoring in a real time scenarios camera device should be able to detect the location, accuracy of time and car registration number for investigation of traffic accidents. However, temperature will not be required in this scenario. For environment monitoring in an IoT system, the information captured by the scalar MIoT device should be abstracted to location, time and temperature (e.g., Plymouth, 11:30, sunny). On the other hand, a number of devices deployed in a real IoT environment require more bandwidth and power to process a video capturing task and send to the control server compare to traditional scalar data. But recently, multimedia processing and storage have been moved to the cloud, in this scenario virtual objects play an important role to complete the task and solve the

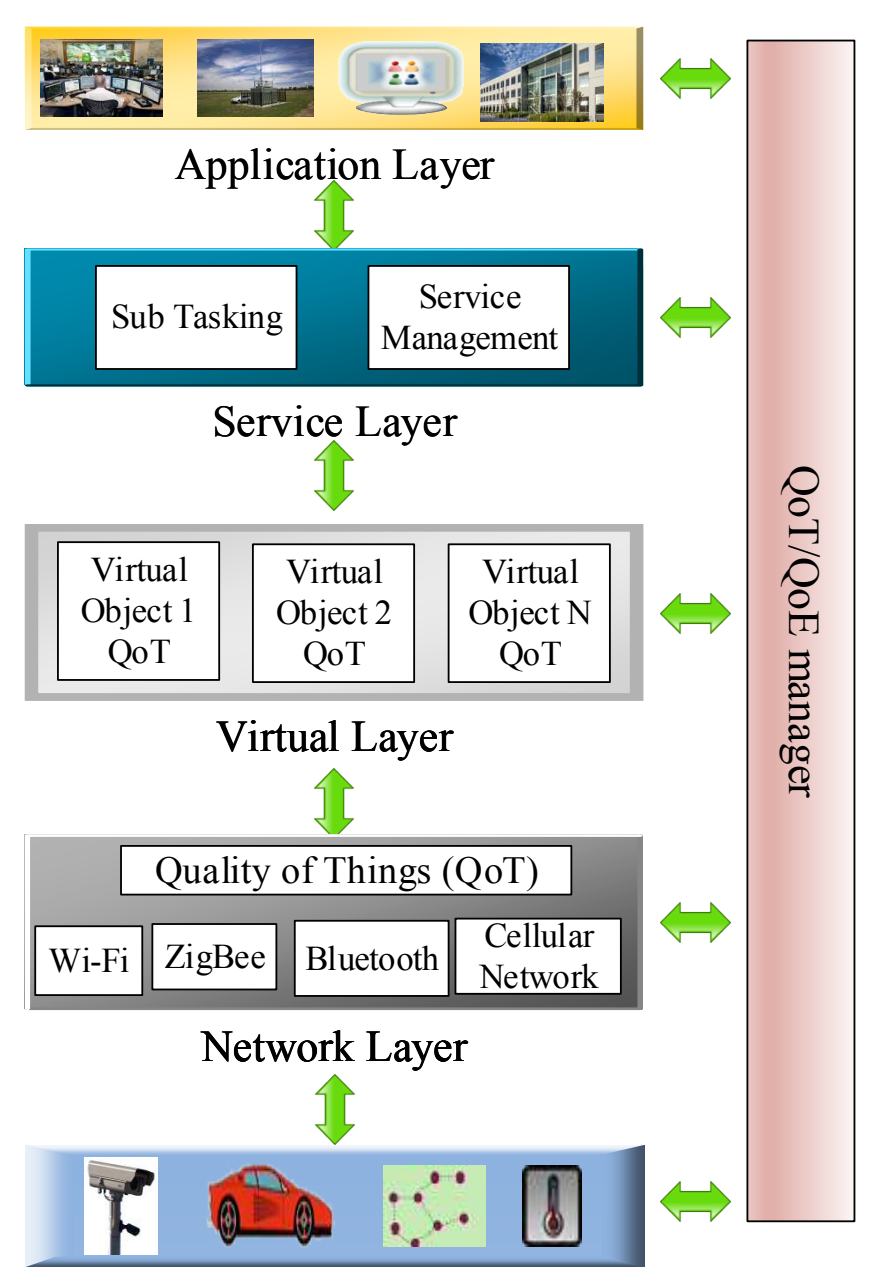

\section{Physical Layer}

Fig. 3. proposed QoT model based on cross layered architecture

device side limitations such as processing and energy. We discuss the virtual layer in the following sections.

2) Network Laye: network layer is the second lower level layer in our model. It collects all the information provided by the heterogeneous MIoT devices and send it to the virtual layer for further processing through different networks, such as Blue-tooth, WI-FI and cellular. It takes into consideration traditional QoS parameters such as packet loss, delay and jitter to transfer the data to upper layers.

3) Virtual Layer: virtual layer is the middle level layer. It creates virtual representation of physical objects/devices and act as a mediator between physical and digital worlds which provides a semantic description of realworld physical objects. Once the physical object registered its functionalities in an IoT environment, the virtual layer creates its virtual object with a virtual template which can be related to one or more realworld devices such as physical devices. The virtual layer is able to solve the issues of heterogeneous devices 
intelligence and scalability issues. This layer also takes care of managing QoT provided by things/objects in the physical layer. Once the physical object joins the networks it needs to be intelligent to be able to react quickly according to the situations. Semantic technologies are the main solution to solve this problem. It takes into consideration virtual object services, resources, and quality factors provided by the physical object. If a new object is detected in an environment it automatically creates its virtual object, and is also very useful to search and find the virtual objects in search engine that are most suitable to complete the application task which is also a semantic technology.

As seen in Fig. 3, each virtual object is assigned a QoT (QoT(VO)) in order to meet the requirement of its associated MIoT device. In (1), the QoT is evaluated by considering QoS factors (QoS), environmental factors (env) and device factors (dev).

$$
Q o T(V O)=f(e n v, \operatorname{dev}, Q o S)
$$

Currently there is no reference on how to measure QoT therefore the objective metrics are used to define QoT.

4) Service Layer: service layer is responsible for managing and analysing the services requested by various applications and is able to provide automatic service. If a service request is received the service analyser divides into smaller sub tasks for a suitable virtual object to fulfil this service task. If a suitable virtual object is not found by semantic algorithm, an approximate or alternative virtual object can fulfil the requirement. Similarly, if the same service is not available all the time, a closely related service can do the service task. Once the query is received, it asks for the data retrieval according to a situation so that the information can be reusable for the same input data, thus it reduces the burden on the virtualization layer.

5) Application Layer: this is the top layer in our model where various applications are responsible for final presentation and processing results. Application factors (App) such as application type, codec type and application requirements. It adopts multimedia codec according to the application type and current device state to meet the requirement of a MIoT device. Therefore, the final QoT(App) in (2) is provided by things and can be expressed as,

$$
Q o T(A p p)=f(Q o T(V O), A p p)
$$

6) QoT/QoE manager: quality management is very challenging in MIoT scenarios because of mobility and heterogeneity related issues. The adoption of virtual objects addresses the above problems. However a QoT/QoE manager is needed to support $\mathrm{M} 2 \mathrm{M}$ and $\mathrm{M} 2 \mathrm{H}$ applications to provide quality services or applications. By considering (3)and (4) QoE factors that influence QoE for $\mathrm{M} 2 \mathrm{H}$ applications are user factors such as end user device on which application is accessed (end user device factors), state of mind when using the application (state of mind factors), user comfort (end user satisfaction factors) and background factors, which can be considered to provide users QoE for $\mathrm{M} 2 \mathrm{H}$ applications where human beings are playing an important role. For example in navigation system applications and E-health monitoring applications. We define QoE for $\mathrm{M} 2 \mathrm{H}$ applications as,

$$
\begin{gathered}
Q o E=f(\text { dev, App, enduser }) \\
Q o T=f(Q o T(A p p))
\end{gathered}
$$

Where QoE factors are factors in $\mathrm{M} 2 \mathrm{H}$ applications and humans are playing the major part. As our future work, we will evaluate QoT and how these influence factors affect the quality for $\mathrm{M} 2 \mathrm{H}$ using subjective experiments.

\section{Future Research DiRections}

The following future research directions of MIoT are observed below.

1) As we discussed in the above sections, current research on multimedia communications in IoT is mainly focused on QoS/QoE metrics to increase end-user satisfaction; however, in IoT systems especially for M2M communications traditional QoE models are not applicable. In this case QoT is more appropriate for such applications. In future IoT systems, an acceptable QoT should be considered to understand the quality by Things.

2) Multimedia monitoring in real time environment requires high processing power and consumes high energy. Traditionally, multimedia devices are battery operated and may not last long enough to complete the task due to the nature of multimedia data. Thus, energy consumption mechanisms such as energy harvesting or scavenging techniques need to be considered to prolong the network lifetime.

3) Future MIoT will be equipped with more multimedia devices, more users access multimedia content from different devices, therefore, how to provide resource allocation to various users is still an issue.

4) Traditionally, QoE is normally measured by using Mean Opinion Score $(\mathrm{MoS})$, future research should find the way on how to measure QoT for M2M applications.

5) Security and privacy is an important issue when transmitting multimedia data to the cloud. Attacks on things will breach the privacy of the IoT system. Further research is needed to protect things privacy while maintaining acceptable QoT for MIoT applications.

\section{CONCLUSION}

This paper has introduced the new concept of QoT for IoT systems based on the MIoT. MIoT and its enabling technologies were discussed and QoT factors were outlined. The QoT model based on cross layer architecture which can provide better quality by things according to the current device state was also proposed in this paper. Finally, this work 
presented future research directions of QoT and MIoT. As our future work, we will device QoT measurement methodology for $\mathrm{M} 2 \mathrm{M}$ and $\mathrm{M} 2 \mathrm{H}$ applications.

\section{REFERENCES}

[1] Statista. Internet of things (iot): number of connected devices worldwide from 2012 to 2020 (in billions). [Online]. Available: https://www.statista.com/statistics/471264/iot-numberof-connected-devices-worldwide/.

[2] L. Atzori, A. Iera, and G. Morabito, "The internet of things: A survey," Computer networks, vol. 54, no. 15, pp. 2787-2805, 2010.

[3] Cisco. (2016) Cisco visual networking index: Forecast and methodology, 2015-2020,. [Online]. Available: http://www.cisco.com /c/en/us/solutions/collateral/service-provider/visual-networking-indexvni/complete-white-paper-c11-481360.html

[4] S. A. Alvi, B. Afzal, G. A. Shah, L. Atzori, and W. Mahmood, "Internet of multimedia things: Vision and challenges," Ad Hoc Networks, vol. 33, pp. 87-111, 2015.

[5] R. Yao, W. Wang, M. Farrokh-Baroughi, H. Wang, and Y. Qian, "Quality-driven energy-neutralized power and relay selection for smart grid wireless multimedia sensor based iots," IEEE Sensors Journal, vol. 13, no. 10, pp. 3637-3644, 2013.

[6] K. Brunnström, S. A. Beker, K. De Moor, A. Dooms, S. Egger, M.-N. Garcia, T. Hossfeld, S. Jumisko-Pyykkö, C. Keimel, M.-C. Larabi et al., "Qualinet white paper on definitions of quality of experience," 2013.

[7] R. Duan, X. Chen, and T. Xing, "A qos architecture for iot," in Internet of Things (iThings/CPSCom), 2011 International Conference on and 4th International Conference on Cyber, Physical and Social Computing. IEEE, 2011, pp. 717-720.

[8] A. Floris and L. Atzori, "Quality of experience in the multimedia internet of things: Definition and practical use-cases," in Communication Workshop (ICCW), 2015 IEEE International Conference on. IEEE, 2015, pp. 1747-1752.

[9] B.-W. Chen, W. Ji, F. Jiang, and S. Rho, "Qoe-enabled big video streaming for large-scale heterogeneous clients and networks in smart cities," IEEE Access, vol. 4, pp. 97-107, 2016.

[10] V. De Silva, X. Shi, B. Evans, J. P. Trufero, and G. Peters, "Designing a qoe aware intelligent home gateway for future smart homes," in Future Network and Mobile Summit (FutureNetworkSummit), 2013. IEEE, 2013, pp. 1-8.

[11] V. Pilloni, A. Floris, A. Meloni, and L. Atzori, "Smart home energy management including renewable sources: A qoe-driven approach," IEEE Transactions on Smart Grid, 2016.

[12] H. Wang, Y. Qian, and H. Sharif, "Multimedia communications over cognitive radio networks for smart grid applications," IEEE wireless communications, vol. 20, no. 4, pp. 125-132, 2013.

[13] P. Garcia Lopez, A. Montresor, D. Epema, A. Datta, T. Higashino, A. Iamnitchi, M. Barcellos, P. Felber, and E. Riviere, "Edge-centric computing: Vision and challenges," ACM SIGCOMM Computer Communication Review, vol. 45, no. 5, pp. 37-42, 2015.

[14] V. Gazis, "A survey of standards for machine to machine (m2m) and the internet of things (iot)," IEEE Communications Surveys \& Tutorials, 2016.

[15] L. Tan and N. Wang, "Future internet: The internet of things," in Advanced Computer Theory and Engineering (ICACTE), 2010 3rd International Conference on, vol. 5. IEEE, 2010, pp. V5-376.

[16] Q. Wu, G. Ding, Y. Xu, S. Feng, Z. Du, J. Wang, and K. Long, "Cognitive internet of things: a new paradigm beyond connection," IEEE Internet of Things Journal, vol. 1, no. 2, pp. 129-143, 2014.

[17] M. O. Farooq, T. Kunz, and M. St-Hilaire, "Cross layer architecture for supporting multiple applications in wireless multimedia sensor networks," in Wireless Communications and Mobile Computing Conference (IWCMC), 2011 7th International. IEEE, 2011, pp. 388-393.

[18] D. G. Costa and L. A. Guedes, "A survey on multimedia-based crosslayer optimization in visual sensor networks," Sensors, vol. 11, no. 5, pp. 5439-5468, 2011 